\title{
Copper-Catalyzed Asymmetric [4+1] Cycloadditions of Enones with Diazo Compounds to Form Dihydrofurans
}

\author{
Sunghee Son and Gregory C. Fu* \\ Department of Chemistry, Massachusetts Institute of Technology \\ Cambridge, Massachusetts, 02139 \\ Supporting Information
}

\section{General}

All reactions were carried out in oven-dried glassware under an atmosphere of nitrogen. $\mathrm{CH}_{2} \mathrm{Cl}_{2}$ was purified by passage through a neutral alumina column under argon. CuOTf $0.5 \mathrm{C}_{6} \mathrm{H}_{5} \mathrm{CH}_{3}$ was purchased from Aldrich, and the diazo esters ${ }^{1}$ and $\mathrm{bpy}^{\star 2}$ were prepared according to literature procedures. All other chemicals were purchased from commercial suppliers and used as received, unless noted otherwise.

HPLC analyses were carried out on an Agilent 1100 Series system with Daicel Chiralpak ${ }^{\circledR}$ columns in hexane/isopropanol mixtures. Melting points were measured on a Hoover melting point apparatus and are uncorrected.

\section{Preparation of Starting Material}

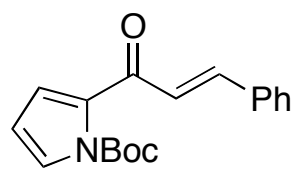

tert-Butyl 2-((E)-3-phenylacryloyl)-1H-pyrrole-1-carboxylate. A 100-mL flask was charged with 2-acetylpyrrole ( $3.27 \mathrm{~g}, 30.0 \mathrm{mmol}), 4$-(dimethylamino)pyridine $(73 \mathrm{mg}$, $0.60 \mathrm{mmol}), \mathrm{NEt}_{3}(4.2 \mathrm{~mL}, 30 \mathrm{mmol})$, and THF $(30 \mathrm{~mL})$. To this mixture was added a solution of Boc anhydride (dropwise; $30 \mathrm{~mL}, 1.0 \mathrm{M}$ in THF, $30.0 \mathrm{mmol}$ ). The reaction mixture was stirred overnight, and then a saturated solution of $\mathrm{NaHCO}_{3}(30 \mathrm{~mL})$ was added. The resulting mixture was extracted with $\mathrm{Et}_{2} \mathrm{O}(30 \mathrm{~mL} \times 3)$, and the combined extracts were dried over $\mathrm{MgSO}_{4}$ and concentrated. The product was purified by column chromatography (hexanes/EtOAc 5:1), which furnished $5.64 \mathrm{~g}(90 \%)$ of N-Boc-2acetylpyrrole.

$\mathrm{N}$-Boc-2-acetylpyrrole $(5.64 \mathrm{~g}, 27.0 \mathrm{mmol})$ was added to a stirred solution of LiHMDS (1.0 M in THF; $28 \mathrm{~mL}, 28 \mathrm{mmol}$ ) at $-78^{\circ} \mathrm{C}$ under argon, and the resulting mixture was stirred for $2 \mathrm{~h}$. Then, benzaldehyde $(2.8 \mathrm{~mL}, 27 \mathrm{mmol})$ was added, and the reaction mixture was stirred for an additional $2 \mathrm{~h}$. The reaction was then quenched by the addition of saturated aq $\mathrm{NH}_{4} \mathrm{Cl}(40 \mathrm{~mL})$, and the mixture was extracted with $\mathrm{Et}_{2} \mathrm{O}$ 
(30 $\mathrm{mL}$ x 3). The combined extracts were dried over $\mathrm{MgSO}_{4}$, concentrated, and purified by column chromatography (hexanes/EtOAc 5:1), which furnished N-Boc-2-(3hydroxy-1-oxo-3-phenylpropyl)pyrrole $(6.82 \mathrm{~g}, 80 \%)$.

$\mathrm{NEt}_{3}(9.0 \mathrm{~mL}, 65 \mathrm{mmol})$ and $\mathrm{MsCl}(1.65 \mathrm{~mL}, 21.7 \mathrm{mmol})$ were added to a solution of $\mathrm{N}$-Boc-2-(3-hydroxy-1-oxo-3-phenylpropyl)pyrrole (6.82 g, $21.7 \mathrm{mmol}$ ) in THF (20 mL). This reaction mixture was stirred overnight, and then it was quenched by the addition of saturated aq $\mathrm{NH}_{4} \mathrm{Cl}(40 \mathrm{~mL})$. The mixture was extracted with $\mathrm{Et}_{2} \mathrm{O}(30 \mathrm{~mL} \times 3)$, and the combined extracts were dried over $\mathrm{MgSO}_{4}$, concentrated, and purified by column chromatography (hexanes/EtOAc 5:1), which furnished tert-butyl 2-((E)-3phenylacryloyl)-1H-pyrrole-1-carboxylate ( $5.89 \mathrm{~g}, 91 \%)$. mp 75-76 ${ }^{\circ} \mathrm{C}$;

${ }^{1} \mathrm{H} \mathrm{NMR}\left(\mathrm{CDCl}_{3}, 400 \mathrm{MHz}\right) \delta 7.66(\mathrm{~d}, J=16.0 \mathrm{~Hz}, 1 \mathrm{H}), 7.60-7.57(\mathrm{~m}, 2 \mathrm{H}), 7.43-7.40$ $(\mathrm{m}, 4 \mathrm{H}), 7.12(\mathrm{~d}, J=16.0 \mathrm{~Hz}, 1 \mathrm{H}), 6.86(\mathrm{dd}, J=3.5,1.6 \mathrm{~Hz}, 1 \mathrm{H}), 6.25(\mathrm{t}, J=3.3 \mathrm{~Hz}, 1 \mathrm{H})$, 1.56 (s, 9H);

${ }^{13} \mathrm{C} \mathrm{NMR}\left(\mathrm{CDCl}_{3}, 100 \mathrm{MHz}\right) \delta 182.8,149.0,143.7,135.0,134.3,130.6,129.1,128.5$, 127.4, 125.7, 120.8, 110.5, 85.2, 27.0;

IR (film) 2981, 1748, 1661, 1639, 1606, 1440, 1415, 1315, $1150 \mathrm{~cm}^{-1}$;

HRMS (ESI) calcd for $\mathrm{C}_{18} \mathrm{H}_{19} \mathrm{NO}_{3} \mathrm{Na}\left(\mathrm{M}+\mathrm{Na}^{+}\right)$320.1257, found 320.1266.

All other materials have been reported previously. 


\section{Copper-Catalyzed Asymmetric [4+1] Cycloadditions (Table 3)}

All yields, dr's, and ee's are the average of two runs, one with (-)-bpy* and one with $(+)$-bpy*. All dr's were determined through analysis of the ${ }^{1} \mathrm{H}$ NMR spectra of the unpurified reaction mixtures.

General Procedure A. The catalyst was prepared by adding a solution of (-)-bpy* ( $8.4 \mathrm{mg}$, $0.013 \mathrm{mmol})$ in $\mathrm{CH}_{2} \mathrm{Cl}_{2}(4 \mathrm{~mL})$ to CuOTf $\cdot 0.5 \mathrm{C}_{6} \mathrm{H}_{5} \mathrm{CH}_{3}(2.6 \mathrm{mg}, 0.010 \mathrm{mmol}$ ) and stirring the resulting mixture for $20 \mathrm{~min}$. This solution was then added to the enone (1.0 $\mathrm{mmol})$, and the mixture was stirred for $5 \mathrm{~min}$. Then, a solution of the diazoacetate (1.1 $\mathrm{mmol})$ in $\mathrm{CH}_{2} \mathrm{Cl}_{2}(1.0 \mathrm{~mL})$ was added. After $1 \mathrm{~h}$ of stirring at room temperature, the reaction mixture was filtered through a plug of silica gel $\left(50 \% \mathrm{Et}_{2} \mathrm{O} /\right.$ hexane as the eluant).

General Procedure B. The catalyst was prepared by adding a solution of (-)-bpy* ( $7.7 \mathrm{mg}, 0.012 \mathrm{mmol})$ in $\mathrm{CH}_{2} \mathrm{Cl}_{2}\left(10 \mathrm{~mL}\right.$ ) to $\mathrm{CuOTf} \cdot 0.5 \mathrm{C}_{6} \mathrm{H}_{5} \mathrm{CH}_{3}(2.6 \mathrm{mg}, 0.010 \mathrm{mmol}$ ) and stirring the resulting mixture for $20 \mathrm{~min}$. This solution was then added to the enone (1.0 mmol) in $\mathrm{CH}_{2} \mathrm{Cl}_{2}(60 \mathrm{~mL})$, and the mixture was stirred for $5 \mathrm{~min}$. Then, a solution of the diazoacetate $(1.4 \mathrm{mmol})$ in $\mathrm{CH}_{2} \mathrm{Cl}_{2}(10 \mathrm{~mL})$ was added dropwise. After $1 \mathrm{~h}$ of stirring at room temperature, the reaction mixture was filtered through a plug of silica gel $(50 \%$ $\mathrm{Et}_{2} \mathrm{O} /$ hexane as the eluant).

(2R,3S)-2,6-Diisopropylphenyl 2,3-dihydro-3,5-diphenylfuran-2-carboxylate (Table 3, entry 1). General Procedure A was followed, with (-)-bpy ${ }^{*}$, trans-chalcone (208 mg, $1.0 \mathrm{mmol})$, and 2,6-diisopropylphenyldiazoacetate $(271 \mathrm{mg}, 1.1 \mathrm{mmol})$. After chromatography on silica gel (hexanes/ ethyl ether 100:1), the title compound was isolated as a colorless oil: run 1, $341 \mathrm{mg}(80 \%$; $86 \%$ de, $84 \%$ ee); run 2, $328 \mathrm{mg}(77 \%$; $86 \%$ de, $86 \%$ ee). The ee was determined on an AD-H column (hexanes/ iso-propanol 99:1, flow $0.7 \mathrm{~mL} / \mathrm{min}$ ) with $\mathrm{t}_{\mathrm{r}}$ (major) $12.2 \mathrm{~min}, \mathrm{t}_{\mathrm{r}}($ minor) $13.0 \mathrm{~min}$.

$[\alpha]^{20}{ }_{D}=-106\left(\mathrm{c}=1.00, \mathrm{CH}_{2} \mathrm{Cl}_{2}\right)$;

${ }^{1} \mathrm{H}$ NMR $\left(\mathrm{C}_{6} \mathrm{D}_{6}, 400 \mathrm{MHz}\right)$ ठ 7.75-7.73 (m, 2H), 7.33-7.31 (m, 2H), 7.18-7.04 (m, 9H), $5.35(\mathrm{~d}, J=2.9 \mathrm{~Hz}, 1 \mathrm{H}), 5.26(\mathrm{~d}, J=4.9 \mathrm{~Hz}, 1 \mathrm{H}), 4.83(\mathrm{dd}, J=4.8,1.8 \mathrm{~Hz}, 1 \mathrm{H}), 3.14$ (septet, $J=6.8 \mathrm{~Hz}, 2 \mathrm{H}), 1.16(\mathrm{~d}, 12 \mathrm{H})$;

${ }^{13} \mathrm{C}$ NMR $\left(\mathrm{C}_{6} \mathrm{D}_{6}, 100 \mathrm{MHz}\right) \delta 170.6,157.4,146.5,143.3,141.1,130.7,129.6,129.0,128.9$, 127.5, 126.4, 124.7, 99.5, 86.6, 54.4, 28.3, 23.6;

IR (film) 3064, 3029, 2965, 2931, 2870, 1774, 1753, 1649, 1602, 1494, 1449, $1213 \mathrm{~cm}^{-1}$;

HRMS (ESI) calcd for $\mathrm{C}_{29} \mathrm{H}_{30} \mathrm{O}_{3} \mathrm{Na}\left(\mathrm{M}+\mathrm{Na}^{+}\right)$449.2087, found 449.2102.

(2R,3S)-2,6-Diisopropylphenyl 2,3-dihydro-3-phenyl-5-(4trifluoromethylphenyl)furan-2-carboxylate (Table 3, entry 2). General Procedure A was followed, with (-)-bpy ${ }^{*}$, trans-4'-(trifluoromethyl)chalcone $(276 \mathrm{mg}, 1.0 \mathrm{mmol})$, and 2,6-diisopropylphenyldiazoacetate $(271 \mathrm{mg}, 1.1 \mathrm{mmol})$. After chromatography on silica gel (hexanes/ethyl ether 100:1), the title compound was isolated as a colorless oil: run 1, 
$282 \mathrm{mg}$ (57\%; 90\% de, 76\% ee); run 2, $297 \mathrm{mg}$ (60\%; 90\% de, 76\% ee). The ee was determined on an AD-H column (hexanes/iso-propanol 99:1, flow $1.0 \mathrm{~mL} / \mathrm{min}$ ) with $\mathrm{t}_{\mathrm{r}}$ (major) $7.8 \mathrm{~min}, \mathrm{t}_{\mathrm{r}}$ (minor) $10.0 \mathrm{~min}$.

$[\alpha]_{D}^{20}=-90.1\left(\mathrm{c}=1.00, \mathrm{CH}_{2} \mathrm{Cl}_{2}\right)$;

${ }^{1} \mathrm{H}$ NMR $\left(\mathrm{C}_{6} \mathrm{D}_{6}, 400 \mathrm{MHz}\right) \delta$ 7.54-7.52 (m, 2H), 7.33-7.27 (m, 4H), 7.18-7.04 (m, 6H), $5.29(\mathrm{~d}, J=2.9 \mathrm{~Hz}, 1 \mathrm{H}), 5.23(\mathrm{~d}, J=4.9 \mathrm{~Hz}, 1 \mathrm{H}), 4.79(\mathrm{dd}, J=4.5,3.1 \mathrm{~Hz}, 1 \mathrm{H}), 3.20-3.00$ $(\mathrm{m}, 2 \mathrm{H}), 1.17(\mathrm{~d}, J=6.9 \mathrm{~Hz}, 12 \mathrm{H})$;

${ }^{13} \mathrm{C}$ NMR $\left(\mathrm{C}_{6} \mathrm{D}_{6}, 100 \mathrm{MHz}\right) \delta$ 170.3, 156.0, 146.3, 142.8, 140.9, 129.7, 128.3, 128.0, 127.6, 126.6, 126.4, 126.0, 125.9, 124.8, 123.7, 101.9, 86.5, 54.4, 28.3, 23.6;

IR (film) 3066, 3030, 2966, 2932, 2872, 1773, 1755, 1647, 1619, 1493, 1457, 1326, 1165, $1070 \mathrm{~cm}^{-1}$;

HRMS (ESI) calcd for $\mathrm{C}_{30} \mathrm{H}_{29} \mathrm{~F}_{3} \mathrm{O}_{3} \mathrm{Na}\left(\mathrm{M}+\mathrm{Na}^{+}\right)$517.1961, found 517.1977.

(2R,3S)-2,6-Diisopropylphenyl 2,3-dihydro-3-phenyl-5-(4-chlorophenyl)furan-2carboxylate (Table 3, entry 3). General Procedure A was followed, with (-)-bpy ${ }^{*}$, trans4'-chlorochalcone (243 mg, $1.0 \mathrm{mmol})$, and 2,6-diisopropylphenyldiazoacetate $(271 \mathrm{mg}$, $1.1 \mathrm{mmol}$ ). After chromatography on silica gel (hexanes/ethyl ether 100:1), the title compound was isolated as a colorless oil: run 1, $360 \mathrm{mg}$ ( $78 \%$; $92 \%$ de, $88 \%$ ee); run 2 , $350 \mathrm{mg}$ (76\%; 88\% de, 87\% ee). The ee was determined on an AD-H column (hexanes / iso-propanol 99:1, flow $1.0 \mathrm{~mL} / \mathrm{min}$ ) with $\mathrm{t}_{\mathrm{r}}$ (major) $6.5 \mathrm{~min}, \mathrm{t}_{\mathrm{r}}$ (minor) $8.3 \mathrm{~min}$.

$[\alpha]^{20}{ }_{D}=-108\left(\mathrm{c}=1.00, \mathrm{CH}_{2} \mathrm{Cl}_{2}\right)$;

${ }^{1} \mathrm{H}$ NMR $\left(\mathrm{C}_{6} \mathrm{D}_{6}, 400 \mathrm{MHz}\right)$ d 7.46-7.43 (m, 2H), 7.30-7.28 (m, 2H), 7.18-7.04 (m, 8H), $5.23(\mathrm{~d}, J=3.3 \mathrm{~Hz}, 1 \mathrm{H}), 5.22(\mathrm{~d}, J=5.1 \mathrm{~Hz}, 1 \mathrm{H}), 4.79(\mathrm{dd}, J=4.8,3.0 \mathrm{~Hz}, 1 \mathrm{H}), 3.10$ (septet, $J=6.4 \mathrm{~Hz}, 2 \mathrm{H}), 1.16(\mathrm{~d}, J=6.9 \mathrm{~Hz}, 12 \mathrm{H})$;

${ }^{13} \mathrm{C}$ NMR $\left(\mathrm{C}_{6} \mathrm{D}_{6}, 100 \mathrm{MHz}\right) \delta 170.4,156.3,146.4,143.1,141.0,135.4,129.6,129.2,129.1$, 128.5, 128.1, 127.7, 127.5, 124.8, 100.1, 86.5, 54.4, 28.3, 23.7;

IR (film) 3065, 3030, 2965, 2931, 2871, 1773, 1755, 1648, 1598, 1490, 1456, $1163 \mathrm{~cm}^{-1}$; HRMS (ESI) calcd for $\mathrm{C}_{29} \mathrm{H}_{29} \mathrm{ClO}_{3} \mathrm{Na}\left(\mathrm{M}+\mathrm{Na}^{+}\right) 483.1697$, found 483.1720 .

\section{(2R,3S)-2,6-Diisopropylphenyl 2-acetoxy-5-(4-methoxyphenyl)-5-oxo-3-} phenylpentanoate (Table 3, entry 4). General Procedure A was followed, with (-)bpy $^{*}$, trans-4'-methoxychalcone $(238 \mathrm{mg}, 1.0 \mathrm{mmol})$, and 2,6diisopropylphenyldiazoacetate $(271 \mathrm{mg}, 1.1 \mathrm{mmol})$. To the unpurified reaction mixture was added $\mathrm{CH}_{3} \mathrm{CN}(2 \mathrm{~mL})$ and $10 \%$ aq $\mathrm{HBF}_{4}(2 \mathrm{~mL})$. The mixture was stirred for $10 \mathrm{~min}$, and then it was diluted with water and extracted with $\mathrm{Et}_{2} \mathrm{O}(20 \mathrm{~mL} \times 3)$. The combined organic layers were washed with water, dried over $\mathrm{Na}_{2} \mathrm{SO}_{4}$, and concentrated. The resulting hydroxyketone was dissolved in $\mathrm{CH}_{2} \mathrm{Cl}_{2}(10 \mathrm{~mL})$, and $\mathrm{NEt}_{3}(0.84 \mathrm{~mL}, 6.0$ mmol), DMAP (24.4 mg, $0.20 \mathrm{mmol})$, and $\mathrm{Ac}_{2} \mathrm{O}(0.47 \mathrm{~mL}, 5.0 \mathrm{mmol})$ were added to the stirred solution. After $15 \mathrm{~min}$, the reaction mixture was diluted with water and extracted with $\mathrm{CH}_{2} \mathrm{Cl}_{2}(20 \mathrm{~mL} \times 3)$. The combined organic layers were washed with brine, dried over $\mathrm{Na}_{2} \mathrm{SO}_{4}$, and concentrated. After chromatography on silica gel (hexanes/ethyl ether 20:1), the title compound was isolated as a white solid: run 1, 360 
mg ( $85 \%$; 90\% de, $92 \%$ ee); run 2, $350 \mathrm{mg}(83 \% ; 90 \%$ de, $91 \%$ ee). The ee was determined on an OD-H column (hexanes/iso-propanol 99:1, flow $1.0 \mathrm{~mL} / \mathrm{min}$ ) with $\mathrm{t}_{\mathrm{r}}$ (major) 17.8 min, $\mathrm{t}_{\mathrm{r}}$ (minor) $26.0 \mathrm{~min}$.

mp 158-161 C;

$[\alpha]^{20}=-58.8\left(\mathrm{c}=1.00, \mathrm{CH}_{2} \mathrm{Cl}_{2}\right)$;

${ }^{1} \mathrm{H}$ NMR $\left(\mathrm{CDCl}_{3}, 400 \mathrm{MHz}\right)$ 8 7.97-7.94 (m, 2H), 7.48-7.46 (m, 2H), 7.35-7.31 (m, 2H), 7.27-7.20 (m, 2H), 7.14-7.13 (m, 2H), 6.95-6.91 (m, 2H), $5.64(\mathrm{~d}, J=3.8 \mathrm{~Hz}, 1 \mathrm{H}), 4.39(\mathrm{dt}, J$ $=10.2,3.5 \mathrm{~Hz}, 1 \mathrm{H}), 3.87(\mathrm{~s}, 3 \mathrm{H}), 3.82(\mathrm{dd}, J=18.0,10.4 \mathrm{~Hz}, 1 \mathrm{H}), 3.40(\mathrm{dd}, J=17.8,3.1 \mathrm{~Hz}$, $1 \mathrm{H}), 3.10-2.80(\mathrm{~m}, 1 \mathrm{H}), 2.80-2.50(\mathrm{~m}, 1 \mathrm{H}), 2.21(\mathrm{~s}, 3 \mathrm{H}), 1.14(\mathrm{~d}, J=6.8 \mathrm{~Hz}, 6 \mathrm{H}), 1.30-0.80$ $(\mathrm{m}, 6 \mathrm{H})$;

${ }^{13} \mathrm{C}$ NMR $\left(\mathrm{C}_{6} \mathrm{D}_{6}, 100 \mathrm{MHz}\right) \delta 195.4,170.3,168.2,163.8,145.1,140.2,130.5,130.0,128.8$, 128.5, 127.6, 127.0, 124.1, 113.9, 76.0, 55.7, 41.8, 38.9, 27.3, 24.0, 22.8, 20.8;

IR (film) 2964, 2929, 2872, 1765, 1749, 1677, 1597, 1510, 1456, $1257 \mathrm{~cm}^{-1}$;

HRMS (ESI) calcd for $\mathrm{C}_{32} \mathrm{H}_{36} \mathrm{O}_{6} \mathrm{Na}\left(\mathrm{M}+\mathrm{Na}^{+}\right)$539.2404, found 539.2396.

(2R,3S)-2,6-Diisopropylphenyl 2,3-dihydro-3-(4-chlorophenyl)-5-phenyl-furan-2carboxylate (Table 3, entry 5). General Procedure A was followed, with (-)-bpy ${ }^{*}$, trans4-chlorochalcone (243 mg, $1.0 \mathrm{mmol})$, and 2,6-diisopropylphenyldiazoacetate $(271 \mathrm{mg}$, $1.1 \mathrm{mmol}$ ). After chromatography on silica gel (hexanes/ ethyl ether 100:1), the title compound was isolated as a colorless oil: run 1, $360 \mathrm{mg}$ (78\%; 90\% de, 88\% ee); run 2, $383 \mathrm{mg}$ (83\%; 94\% de, $88 \%$ ee). The ee was determined on an OD-H column (hexanes/ iso-propanol 99:1, flow $0.9 \mathrm{~mL} / \mathrm{min}$ ) with $\mathrm{t}_{\mathrm{r}}$ (major) $8.1 \mathrm{~min}, \mathrm{t}_{\mathrm{r}}$ (minor) $5.9 \mathrm{~min}$. $[\alpha]^{20}{ }_{D}=-126\left(\mathrm{c}=1.00, \mathrm{CH}_{2} \mathrm{Cl}_{2}\right)$;

${ }^{1} \mathrm{H}$ NMR $\left(\mathrm{C}_{6} \mathrm{D}_{6}, 400 \mathrm{MHz}\right)$ 8 7.74-7.71 (m, 2H), 7.16-7.00 (m, 10H), $5.23(\mathrm{~d}, J=2.9 \mathrm{~Hz}$, $1 \mathrm{H}), 5.11(\mathrm{~d}, J=4.9 \mathrm{~Hz}, 1 \mathrm{H}), 4.69(\mathrm{dd}, J=4.7,3.0 \mathrm{~Hz}, 1 \mathrm{H}), 3.12$ (septet, $2 \mathrm{H}), 1.16(\mathrm{~d}, J=$ $6.9 \mathrm{~Hz}, 12 \mathrm{H})$;

${ }^{13} \mathrm{C}$ NMR $\left(\mathrm{C}_{6} \mathrm{D}_{6}, 100 \mathrm{MHz}\right) \delta 170.4,157.7,146.4,141.7,141.0,133.9,130.5,129.8,129.7$, $129.5,129.0,127.5,126.4,124.8,99.0,86.3,53.6,28.3,23.6$;

IR (film) 3065, 3029, 2965, 2931, 2871, 1775, 1752, 1648, 1601, 1577, 1492, $1163 \mathrm{~cm}^{-1}$; HRMS (ESI) calcd for $\mathrm{C}_{29} \mathrm{H}_{29} \mathrm{ClO}_{3} \mathrm{Na}\left(\mathrm{M}+\mathrm{Na}^{+}\right)$483.1697, found 483.1716.

\section{(2R,3S)-2,6-Diisopropylphenyl 2,3-dihydro-3-(4-methoxyphenyl)-5-phenyl-furan-} 2-carboxylate (Table 3, entry 6). General Procedure A was followed, with (-)-bpy*, trans-4-methoxychalcone (238 $\mathrm{mg}, 1.0 \mathrm{mmol})$, and 2,6-diisopropylphenyldiazoacetate (271 mg, $1.1 \mathrm{mmol}$ ). After chromatography on silica gel (hexanes/ethyl ether 100:1), the title compound was isolated as a colorless oil: run 1, $384 \mathrm{mg}(84 \%$; $80 \%$ de, $93 \%$ ee); run $2,379 \mathrm{mg}$ ( $83 \% ; 80 \%$ de, $93 \%$ ee). The ee was determined on an AD-H column (hexanes/ iso-propanol 99:1, flow $1.0 \mathrm{~mL} / \mathrm{min}$ ) with $\mathrm{t}_{\mathrm{r}}$ (major) $7.4 \mathrm{~min}, \mathrm{t}_{\mathrm{r}}$ (minor) $9.6 \mathrm{~min}$. $[\alpha]^{20}{ }_{D}=-130\left(\mathrm{c}=1.00, \mathrm{CH}_{2} \mathrm{Cl}_{2}\right)$;

${ }^{1} \mathrm{H}$ NMR $\left(\mathrm{C}_{6} \mathrm{D}_{6}, 400 \mathrm{MHz}\right) \delta$ 7.77-7.75 (m, 2H), 7.26-7.24 (m, 2H), 7.16-7.04 (m, 6H), 6.81-6.79 (m, 2H), $5.39(\mathrm{~d}, J=2.9 \mathrm{~Hz}, 1 \mathrm{H}), 5.29(\mathrm{~d}, J=5.0 \mathrm{~Hz}, 1 \mathrm{H}), 4.84(\mathrm{dd}, J=5.0,2.9$ $\mathrm{Hz}, 1 \mathrm{H}), 3.33(\mathrm{~s}, 3 \mathrm{H}), 3.16$ (septet, $J=6.7 \mathrm{~Hz}, 2 \mathrm{H}), 1.18(\mathrm{~d}, J=6.9 \mathrm{~Hz}, 12 \mathrm{H})$; 
${ }^{13} \mathrm{C}$ NMR $\left(\mathrm{C}_{6} \mathrm{D}_{6}, 100 \mathrm{MHz}\right) \delta 170.7,160.0,157.2,146.5,141.1,135.3,130.8,129.6,129.2$, 129.0, 127.5, 126.4, 124.7, 115.0, 99.8, 86.8, 55.2, 53.8, 28.3, 23.7;

IR (film) 3065, 3029, 2965, 2932, 2870, 1773, 1752, 1648, 1610, 1584, 1512, 1462, 1448, $1251,1145,1033 \mathrm{~cm}^{-1}$;

HRMS (ESI) calcd for $\mathrm{C}_{30} \mathrm{H}_{32} \mathrm{O}_{4} \mathrm{Na}\left(\mathrm{M}+\mathrm{Na}^{+}\right)$479.2193, found 479.2216.

tert-Butyl 2-((4S,5R)-5-((2,6-diisopropylphenoxy)carbonyl)-4,5-dihydro-4phenylfuran-2-yl)-1H-pyrrole-1-carboxylate (Table 3, entry 7). General Procedure A was followed, with (-)-bpy*, tert-butyl 2-((E)-3-phenylacryloyl)-1H-pyrrole-1carboxylate (297 mg, $1.0 \mathrm{mmol})$, and 2,6-diisopropylphenyldiazoacetate (271 mg, 1.1 $\mathrm{mmol}$ ). After chromatography on silica gel (hexanes/ethyl ether 100:1), the title compound was isolated as a colorless oil: run 1, $347 \mathrm{mg}(66 \%$; >90\% de, $94 \%$ ee); run 2 , $368 \mathrm{mg}$ (70\%; >90\% de, 92\% ee). The ee was determined on an OD-H column (hexanes/ iso-propanol 99:1, flow $0.9 \mathrm{~mL} / \mathrm{min}$ ) with $\mathrm{t}_{\mathrm{r}}$ (major) $5.2 \mathrm{~min}, \mathrm{t}_{\mathrm{r}}($ minor) $4.8 \mathrm{~min}$.

$[\alpha]^{20}{ }_{D}=-129\left(\mathrm{c}=1.00, \mathrm{CH}_{2} \mathrm{Cl}_{2}\right)$;

${ }^{1} \mathrm{H}$ NMR $\left(\mathrm{C}_{6} \mathrm{D}_{6}, 400 \mathrm{MHz}\right) \delta$ 7.54-7.52 (m, 2H), 7.26 (dd, $\left.J=3.2,1.8 \mathrm{~Hz}, 1 \mathrm{H}\right), 7.21-7.17$ $(\mathrm{m}, 2 \mathrm{H}), 7.11-7.03(\mathrm{~m}, 4 \mathrm{H}), 6.82(\mathrm{dd}, J=3.4,1.8 \mathrm{~Hz}, 1 \mathrm{H}), 6.03(\mathrm{t}, J=3.4 \mathrm{~Hz}, 1 \mathrm{H}), 5.77(\mathrm{~d}, J$ $=2.8 \mathrm{~Hz}, 1 \mathrm{H}), 5.31(\mathrm{~d}, J=5.8 \mathrm{~Hz}, 1 \mathrm{H}), 4.90(\mathrm{dd}, J=5.8,2.7 \mathrm{~Hz}, 1 \mathrm{H}), 3.13$ (septet, 2H), 1.25 $(\mathrm{s}, 9 \mathrm{H}), 1.15(\mathrm{~d}, J=6.8 \mathrm{~Hz}, 12 \mathrm{H})$;

${ }^{13} \mathrm{C}$ NMR $\left(\mathrm{C}_{6} \mathrm{D}_{6}, 100 \mathrm{MHz}\right) \delta 170.6,150.3,149.0,146.5,143.5,141.1,129.5,128.5,128.0$, 127.4, 125.6, 124.8, 124.7, 117.9, 111.3, 103.4, 86.3, 84.0, 55.0, 28.2, 28.0, 23.7;

IR (film) 2966, 2932, 2871, 1754, 1652, 1456, 1306, $1144 \mathrm{~cm}^{-1}$; HRMS (ESI) calcd for $\mathrm{C}_{32} \mathrm{H}_{37} \mathrm{NO}_{5} \mathrm{Na}\left(\mathrm{M}+\mathrm{Na}^{+}\right)$538.2564, found 538.2554.

(2R,3S)-2,6-Diisopropylphenyl 3-(furan-3-yl)-2,3-dihydro-5-phenylfuran-2carboxylate (Table 3, entry 8). General Procedure A was followed, with (-)-bpy*, (E)-1(3-furyl)-3-phenyl-2-propen-1-one (198 mg, $1.0 \mathrm{mmol}$ ), and 2,6-diisopropylphenyldiazoacetate ( $271 \mathrm{mg}, 1.1 \mathrm{mmol}$ ). After chromatography on silica gel (hexanes/ethyl ether 100:1), the title compound was isolated as a colorless oil: run 1, $258 \mathrm{mg}(62 \% ; 68 \%$ de, $85 \%$ ee); run 2, $262 \mathrm{mg}(63 \% ; 70 \%$ de, $88 \%$ ee). The ee was determined on an AD-H column (hexanes/iso-propanol 99:1, flow $0.7 \mathrm{~mL} / \mathrm{min}$ ) with $\mathrm{t}_{\mathrm{r}}$ (major) $12.5 \mathrm{~min}, \mathrm{t}_{\mathrm{r}}$ (minor) $13.1 \mathrm{~min}$.

$[\alpha]^{20}{ }_{D}=-97.1\left(\mathrm{c}=1.00, \mathrm{CH}_{2} \mathrm{Cl}_{2}\right)$;

${ }^{1} \mathrm{H}$ NMR $\left(\mathrm{C}_{6} \mathrm{D}_{6}, 400 \mathrm{MHz}\right) \delta$ 7.72-7.70 (m, 2H), 7.14-7.00 (m, 8H), $6.23(\mathrm{~s}, 1 \mathrm{H}), 5.27(\mathrm{~d}, J$ $=2.9 \mathrm{~Hz}, 1 \mathrm{H}), 5.21(\mathrm{~d}, J=5.3 \mathrm{~Hz}, 1 \mathrm{H}), 4.73(\mathrm{dd}, J=5.3,2.8 \mathrm{~Hz}, 1 \mathrm{H}), 3.12($ septet, $2 \mathrm{H}), 1.16$ $(\mathrm{d}, J=6.9 \mathrm{~Hz}, 12 \mathrm{H})$;

${ }^{13} \mathrm{C}$ NMR $\left(\mathrm{C}_{6} \mathrm{D}_{6}, 100 \mathrm{MHz}\right) \delta 170.4,157.2,146.4,144.4,141.0,139.9,130.7,129.6,129.0$, 127.5, 127.3, 126.3, 124.8, 110.1, 98.5, 85.6, 45.0, 28.3, 23.7;

IR (film) 3065, 2965, 2871, 1774, 1753, 1650, 1578, 1448, $1162 \mathrm{~cm}^{-1}$;

HRMS (ESI) calcd for $\mathrm{C}_{27} \mathrm{H}_{28} \mathrm{O}_{4} \mathrm{Na}\left(\mathrm{M}+\mathrm{Na}^{+}\right)$439.1880, found 439.1870 . 
(2R,3R)-2,6-Diisopropylphenyl 2,3-dihydro-5-phenyl-3-(E)-styrylfuran-2carboxylate (Table 3, entry 9). General Procedure A was followed, with (-)-bpy*, trans, trans-1,5-diphenylpenta-2,4-dien-1-one (234 mg, $1.0 \mathrm{mmol})$, and 2,6-

diisopropylphenyldiazo-acetate $(271 \mathrm{mg}, 1.1 \mathrm{mmol})$. After chromatography on silica gel (hexanes/ethyl ether 100:1), the title compound was isolated as a colorless oil: run 1, 344 mg (76\%; 76\% de, 93\% ee); run 2, $344 \mathrm{mg}$ (76\%; 74\% de, 92\% ee). The ee was determined on an AD-H column (hexanes/iso-propanol 99:1, flow $0.7 \mathrm{~mL} / \mathrm{min}$ ) with $\mathrm{t}_{\mathrm{r}}$ (major) 16.8 $\min , \mathrm{t}_{\mathrm{r}}$ (minor) $22.4 \mathrm{~min}$.

$[\alpha]^{20}=-176\left(\mathrm{c}=1.00, \mathrm{CH}_{2} \mathrm{Cl}_{2}\right)$;

${ }^{1} \mathrm{H}$ NMR $\left(\mathrm{C}_{6} \mathrm{D}_{6}, 400 \mathrm{MHz}\right) \delta$ 7.76-7.73 (m, 2H), 7.22-7.20 (m, 2H), 7.16-7.04 (m, 9H), $6.52(\mathrm{~d}, J=15.7 \mathrm{~Hz}, 1 \mathrm{H}), 6.19(\mathrm{dd}, J=15.7,8.3 \mathrm{~Hz}, 1 \mathrm{H}), 5.25(\mathrm{~d}, J=2.8 \mathrm{~Hz}, 1 \mathrm{H}), 5.15(\mathrm{~d}, J$ $=5.5 \mathrm{~Hz}, 1 \mathrm{H}), 4.43(\mathrm{ddd}, J=8.0,5.4,2.6 \mathrm{~Hz}, 1 \mathrm{H}), 3.18$ (septet, $J=6.7 \mathrm{~Hz}, 2 \mathrm{H}), 1.18(\mathrm{~d}, J=$ $6.9 \mathrm{~Hz}, 12 \mathrm{H})$;

${ }^{13} \mathrm{C}$ NMR $\left(\mathrm{C}_{6} \mathrm{D}_{6}, 100 \mathrm{MHz}\right) \delta 170.4,157.2,146.4,141.0,137.4,132.3,130.8,130.2,129.6$, 129.2, 129.0, 128.3, 127.5, 127.2, 126.3, 124.7, 98.4, 84.4, 52.4, 28.3, 23.8;

IR (film) 3061, 3027, 2965, 2930, 2870, 1774, 1752, 1645, 1448, 1163, $1061 \mathrm{~cm}^{-1}$;

HRMS (ESI) calcd for $\mathrm{C}_{31} \mathrm{H}_{32} \mathrm{O}_{3} \mathrm{Na}\left(\mathrm{M}+\mathrm{Na}^{+}\right)$475.2243, found 475.2258.

(2R,3R)-2,6-Diisopropylphenyl 3-butyl-2,3-dihydro-5-phenylfuran-2-carboxylate (Table 3, entry 10). General Procedure A was followed, with (-)-bpy*, (E)-1-phenyl-2hepten-1-one (188 mg, $1.0 \mathrm{mmol})$, and 2,6-diisopropylphenyldiazoacetate $(271 \mathrm{mg}, 1.1$ $\mathrm{mmol}$ ). After chromatography on silica gel (hexanes/ethyl ether 100:1), the title compound was isolated as a colorless oil: run 1, $370 \mathrm{mg}$ (91\%; $96 \%$ de, 79\% ee); run 2, $378 \mathrm{mg}(93 \% ; 97 \%$ de, 76\% ee). The ee was determined on an AD-H column (hexanes/iso-propanol 99:1, flow $1.0 \mathrm{~mL} / \mathrm{min}$ ) with $\mathrm{t}_{\mathrm{r}}$ (major) $4.1 \mathrm{~min}, \mathrm{t}_{\mathrm{r}}$ (minor) $4.9 \mathrm{~min}$. $[\alpha]_{D}^{20}=-53.6\left(\mathrm{c}=1.00, \mathrm{CH}_{2} \mathrm{Cl}_{2}\right)$;

${ }^{1} \mathrm{H}$ NMR $\left(\mathrm{C}_{6} \mathrm{D}_{6}, 400 \mathrm{MHz}\right) \delta$ 7.74-7.72 (m, 2H), 7.14-7.03 (m, 6H), $5.27(\mathrm{~d}, J=2.8 \mathrm{~Hz}$, $1 \mathrm{H}), 5.29(\mathrm{~d}, J=5.3 \mathrm{~Hz}, 1 \mathrm{H}), 3.61-3.52(\mathrm{~m}, 1 \mathrm{H}), 3.23-3.06(\mathrm{~m}, 2 \mathrm{H}), 1.58-1.38(\mathrm{~m}, 2 \mathrm{H}), 1.34-$ $1.10(\mathrm{~m}, 16 \mathrm{H}), 0.85(\mathrm{t}, J=7.1 \mathrm{~Hz}, 3 \mathrm{H})$;

${ }^{13} \mathrm{C}$ NMR $\left(\mathrm{C}_{6} \mathrm{D}_{6}, 100 \mathrm{MHz}\right) \delta 170.9,156.4,146.5,141.1,131.2,129.3,128.9,127.7,127.4$, 126.2, 124.7, 99.4, 84.2, 49.3, 36.1, 29.8, 28.3, 23.8, 23.3, 14.6;

IR (film) 3066, 3029, 2963, 2930, 2871, 1776, 1751, 1650, 1602, 1578, 1466, $1448 \mathrm{~cm}^{-1}$; HRMS (ESI) calcd for $\mathrm{C}_{27} \mathrm{H}_{34} \mathrm{O}_{3} \mathrm{Na}\left(\mathrm{M}+\mathrm{Na}^{+}\right)$429.2400, found 429.2390.

(2R,3S)-2,6-Diisopropylphenyl 5-hexyl-2,3-dihydro-3-phenylfuran-2-carboxylate (Table 3, entry 11). General Procedure B was followed, with (-)-bpy*, (E)-1-phenyl-1nonen-3-one (216 mg, $1.0 \mathrm{mmol})$, and 2,6-diisopropylphenyldiazoacetate (345 mg, 1.4 $\mathrm{mmol}$ ). After chromatography on silica gel (hexanes/ethyl ether 100:1), the title compound was isolated as a colorless oil: run 1, $300 \mathrm{mg}(69 \% ; 84 \%$ de, 77\% ee); run 2, $300 \mathrm{mg}$ (69\%; 88\% de, 73\% ee). The ee was determined after reduction by LAH on an OD-H column (hexanes/iso-propanol 98:2, flow $1.0 \mathrm{~mL} / \mathrm{min}$ ) with $\mathrm{t}_{\mathrm{r}}$ (major) $17.1 \mathrm{~min}$, $\mathrm{t}_{\mathrm{r}}$ (minor) $12.3 \mathrm{~min}$. 
$[\alpha]^{20}{ }_{D}=-174\left(\mathrm{c}=1.00, \mathrm{CH}_{2} \mathrm{Cl}_{2}\right)$;

${ }^{1} \mathrm{H}$ NMR $\left(\mathrm{C}_{6} \mathrm{D}_{6}, 400 \mathrm{MHz}\right)$ ठ 7.38-7.36 (m, 2H), 7.21-7.18 (m, 2H), 7.13-7.04 (m, 4H), $5.16(\mathrm{~d}, J=4.9 \mathrm{~Hz}, 1 \mathrm{H}), 4.71(\mathrm{~d}, J=2.3 \mathrm{~Hz}, 1 \mathrm{H}), 4.68(\mathrm{dd}, J=4.6,2.4 \mathrm{~Hz}, 1 \mathrm{H}), 3.13$ (septet, $J=6.7 \mathrm{~Hz}, 2 \mathrm{H}), 2.25(\mathrm{t}, J=7.5 \mathrm{~Hz}, 2 \mathrm{H}), 1.60$ (quintet, $J=7.5 \mathrm{~Hz}, 2 \mathrm{H}), 1.33-1.10(\mathrm{~m}, 18 \mathrm{H})$, $0.86(\mathrm{t}, J=6.9 \mathrm{~Hz}, 3 \mathrm{H})$;

${ }^{13} \mathrm{C}$ NMR $\left(\mathrm{C}_{6} \mathrm{D}_{6}, 100 \mathrm{MHz}\right) \delta 170.8,161.1,146.4,144.2,141.1,129.5,128.0,127.4,124.7$, 98.6, 86.6, 54.3, 32.3, 29.6, 28.6, 28.3, 27.3, 23.9, 23.3, 14.7;

IR (film) 3065, 3029, 2962, 2930, 2871, 1776, 1754, 1673, 1602, 1493, 1456, 1248, 1144, $1096 \mathrm{~cm}^{-1}$;

HRMS (ESI) calcd for $\mathrm{C}_{29} \mathrm{H}_{38} \mathrm{O}_{3} \mathrm{Na}\left(\mathrm{M}+\mathrm{Na}^{+}\right)$457.2713, found 457.2733.

(2R,3R)-2,6-Diisopropylphenyl 5-hexyl-2,3-dihydro-3-methylfuran-2-carboxylate (Table 3, entry 12). General Procedure B was followed, with (-)-bpy*, (E)-2-decen-4-one (154 mg, $1.0 \mathrm{mmol})$, and 2,6-diisopropylphenyldiazoacetate (345 mg, $1.4 \mathrm{mmol})$. After chromatography on silica gel (hexanes/ ethyl ether 100:1), the title compound was isolated as a colorless oil: run 1, $291 \mathrm{mg}$ (78\%; >99\% de, 72\% ee); run 2, $305 \mathrm{mg}$ (82\%; $>90 \%$ de, $69 \%$ ee). The ee was determined, after reduction by LAH, on an OD-H column (hexanes/iso-propanol 99:1, flow $1.0 \mathrm{~mL} / \mathrm{min}$ ) with $\mathrm{t}_{\mathrm{r}}$ (major) $7.4 \mathrm{~min}, \mathrm{t}_{\mathrm{r}}$ (minor) $8.8 \mathrm{~min}$.

$[\alpha]_{D}^{20}=-70.8\left(\mathrm{c}=1.00, \mathrm{CH}_{2} \mathrm{Cl}_{2}\right)$;

${ }^{1} \mathrm{H}$ NMR $\left(\mathrm{C}_{6} \mathrm{D}_{6}, 400 \mathrm{MHz}\right) \delta$ 7.11-7.04 (m, 3H), $4.77(\mathrm{~d}, J=5.7 \mathrm{~Hz}, 1 \mathrm{H}), 4.52-4.50(\mathrm{~m}$, $1 \mathrm{H}), 3.48-3.45(\mathrm{~m}, 1 \mathrm{H}), 3.13$ (septet, $J=6.8 \mathrm{~Hz}, 2 \mathrm{H}), 2.17(\mathrm{t}, J=7.4 \mathrm{~Hz}, 2 \mathrm{H}), 1.55$ (quintet, $J$ $=7.4 \mathrm{~Hz}, 2 \mathrm{H}), 1.33-1.14(\mathrm{~m}, 18 \mathrm{H}), 1.13-1.10(\mathrm{~d}, J=6.8 \mathrm{~Hz}, 3 \mathrm{H}), 0.84(\mathrm{t}, J=6.7 \mathrm{~Hz}, 3 \mathrm{H})$;

${ }^{13} \mathrm{C}$ NMR $\left(\mathrm{C}_{6} \mathrm{D}_{6}, 100 \mathrm{MHz}\right) \delta 171.0,159.5,146.4,141.1,127.3,124.7,100.1,85.6,43.8$, 32.3, 29.5, 28.6, 28.3, 27.2, 23.8, 23.3, 22.0, 14.6;

IR (film) 3067, 2963, 2930, 2871, 1778, 1751, 1674, 1459, 1245, 1161, $1095 \mathrm{~cm}^{-1}$; HRMS (ESI) calcd for $\mathrm{C}_{24} \mathrm{H}_{36} \mathrm{O}_{3} \mathrm{Na}\left(\mathrm{M}+\mathrm{Na}^{+}\right)$395.2557, found 395.2562. 


\section{Derivatization of Dihydrofurans (eq 2 and eq 3)}

((2R,3S)-2,3-Dihydro-5-hexyl-3-phenylfuran-2-yl)methanol (eq 2). A solution of $\mathrm{LiAlH}_{4}\left(1.0 \mathrm{M}\right.$ in Et $\left.\mathrm{O}_{2} ; 0.23 \mathrm{~mL}, 0.23 \mathrm{mmol}\right)$ was added dropwise to a stirred solution of the ester (run 1, >90\% de and 77\% ee; run 2, >90\% de and $73 \%$ ee; $100 \mathrm{mg}, 0.23 \mathrm{mmol}$ ) in $\mathrm{Et}_{2} \mathrm{O}(5.0 \mathrm{~mL})$. The reaction mixture was stirred for $10 \mathrm{~min}$, and then the reaction was quenched with $1.0 \mathrm{~N} \mathrm{NaOH}(0.2 \mathrm{~mL})$. The mixture was stirred for $5 \mathrm{~min}$, and then it was filtered and concentrated. After chromatography on silica gel (hexanes/ethyl ether 15:1), the title compound was isolated as a colorless oil: run 1, $57 \mathrm{mg}(95 \% ; 77 \%$ ee); run $2,58 \mathrm{mg}(97 \%$; $73 \%$ ee). The ee was determined on an OD-H column (hexanes / isopropanol 98:2, flow $1.0 \mathrm{~mL} / \mathrm{min}$ ) with $\mathrm{t}_{\mathrm{r}}$ (major) $17.1 \mathrm{~min}, \mathrm{t}_{\mathrm{r}}$ (minor) $12.3 \mathrm{~min}$.

$[\alpha]_{D}^{20}=-74.9\left(\mathrm{c}=1.00, \mathrm{CH}_{2} \mathrm{Cl}_{2}\right)$;

${ }^{1} \mathrm{H}$ NMR $\left(\mathrm{C}_{6} \mathrm{D}_{6}, 400 \mathrm{MHz}\right) \delta$ 7.18-7.12 (m, 4H), 7.07-7.04 (m, 1H), $4.56(\mathrm{dt}, J=2.2,1.1$ $\mathrm{Hz}, 1 \mathrm{H}), 4.34(\mathrm{dt}, J=6.7,5.0 \mathrm{~Hz}, 1 \mathrm{H}), 3.83(\mathrm{dq}, J=6.7,1.9 \mathrm{~Hz}, 1 \mathrm{H}), 3.53(\mathrm{t}, J=5.0 \mathrm{~Hz}, 2 \mathrm{H})$, $2.12(\mathrm{t}, J=7.8 \mathrm{~Hz}, 2 \mathrm{H}), 1.71(\mathrm{t}, J=5.6 \mathrm{~Hz}, 1 \mathrm{H}), 1.51$ (quintet, $J=7.5 \mathrm{~Hz}, 2 \mathrm{H}), 1.31-1.17(\mathrm{~m}$, $6 \mathrm{H}), 0.86(\mathrm{t}, J=6.8 \mathrm{~Hz}, 3 \mathrm{H})$;

${ }^{13} \mathrm{C}$ NMR $\left(\mathrm{C}_{6} \mathrm{D}_{6}, 100 \mathrm{MHz}\right) \delta 159.9,145.4,129.2,128.2,127.3,99.4,90.8,64.9,51.6,32.3$, 29.7, 28.7, 27.4, 23.3, 14.6;

IR (film) 3424, 3062, 3028, 2954, 2929, 2859, 1669, 1602, 1493, 1455, $1159 \mathrm{~cm}^{-1}$;

HRMS (ESI) calcd for $\mathrm{C}_{17} \mathrm{H}_{24} \mathrm{O}_{2} \mathrm{Na}\left(\mathrm{M}+\mathrm{Na}^{+}\right)$283.1669, found 283.1676 .

(2R,3R)-2,6-Diisopropylphenyl 2-acetoxy-3-butyl-5-oxo-5-phenylpentanoate (eq 3). The procedure for entry 4 of Table 3 was followed, with the ester (run 1, >90\% de and $79 \%$ ee; run 2, >90\% de and $76 \%$ ee; $50 \mathrm{mg}, 0.12 \mathrm{mmol}), 10 \%$ aq $\mathrm{HBF}_{4}(1.0 \mathrm{~mL}), \mathrm{NEt}_{3}$ $(0.10 \mathrm{~mL}, 0.74 \mathrm{mmol}), \mathrm{DMAP}(3.0 \mathrm{mg}, 0.025 \mathrm{mmol})$, and $\mathrm{Ac}_{2} \mathrm{O}(0.060 \mathrm{~mL}, 0.64 \mathrm{mmol})$. Purification by column chromatography on silica gel (hexanes/ ethyl ether 20:1) furnished the title compound as a colorless oil: run 1, $50 \mathrm{mg}(87 \% ;>90 \%$ de, $78 \%$ ee); run 2, $53 \mathrm{mg}$ (92\%; >90\% de, $76 \%$ ee). The ee was determined on an AD-H column (hexanes/iso-propanol 99:1, flow $1.0 \mathrm{~mL} / \mathrm{min}$ ) with $\mathrm{t}_{\mathrm{r}}$ (major) $5.0 \mathrm{~min}, \mathrm{t}_{\mathrm{r}}$ (minor) $6.3 \mathrm{~min}$.

$[\alpha]^{20}{ }_{D}=-11.4\left(\mathrm{c}=1.00, \mathrm{CH}_{2} \mathrm{Cl}_{2}\right)$;

${ }^{1} \mathrm{H}$ NMR $\left(\mathrm{CDCl}_{3}, 400 \mathrm{MHz}\right) \delta 8.03-8.00(\mathrm{~m}, 2 \mathrm{H}), 7.60-7.57(\mathrm{~m}, 1 \mathrm{H}), 7.50-7.47(\mathrm{~m}, 2 \mathrm{H})$, 7.23-7.13 (m, 3H), $5.54(\mathrm{~d}, J=2.3 \mathrm{~Hz}, 1 \mathrm{H}), 3.24-3.22(\mathrm{~m}, 2 \mathrm{H}), 3.17-3.08(\mathrm{~m}, 1 \mathrm{H}), 3.08-2.90$ $(\mathrm{m}, 1 \mathrm{H}), 2.90-2.60(\mathrm{~m}, 1 \mathrm{H}), 2.22(\mathrm{~s}, 3 \mathrm{H}), 1.62-1.52(\mathrm{~m}, 2 \mathrm{H}), 1.50-1.33(\mathrm{~m}, 4 \mathrm{H}), 1.17(\mathrm{~d}, J=$ $6.3 \mathrm{~Hz}, 12 \mathrm{H}), 0.94(\mathrm{t}, J=6.9 \mathrm{~Hz}, 3 \mathrm{H})$;

${ }^{13} \mathrm{C}$ NMR $\left(\mathrm{C}_{6} \mathrm{D}_{6}, 100 \mathrm{MHz}\right) \delta$ 198.0, 170.6, 169.1, 145.1, 140.6, 137.2, 133.4, 128.9, 128.2, 127.0, 124.1, 73.5, 39.3, 35.4, 31.8, 29.5, 27.4, 23.7, 22.8, 20.8, 14.1;

IR (film) 3065, 2965, 2932, 2871, 1769, 1751, 1689, 1598, 1449, 1366, 1229, $1174 \mathrm{~cm}^{-1}$; HRMS (ESI) calcd for $\mathrm{C}_{29} \mathrm{H}_{38} \mathrm{O}_{5} \mathrm{Na}\left(\mathrm{M}+\mathrm{Na}^{+}\right)$489.2611, found 489.2636. 


\section{Application to Deoxy-C-nucleoside Synthesis (Figure 1)}<smiles>CCCOC=CC(=O)c1ccccc1</smiles>

(E)-3-(2-(Trimethylsilyl)ethoxy)-1-phenylprop-2-en-1-one. 1-Phenylprop-2-yn-1one $(650 \mathrm{mg}, 5.0 \mathrm{mmol})$ was added dropwise to a solution of trimethylsilylethanol (650 $\mathrm{mg} ; 5.5 \mathrm{mmol})$ and $\mathrm{N}$-methylmorpholine $(657 \mathrm{mg}, 6.5 \mathrm{mmol})$ in $\mathrm{CH}_{2} \mathrm{Cl}_{2}(5.0 \mathrm{~mL})$ at r.t. The reaction mixture was stirred for 2 days, and then a saturated solution of $\mathrm{NH}_{4} \mathrm{Cl}(10$ $\mathrm{mL})$ was added. The mixture was extracted with $\mathrm{CH}_{2} \mathrm{Cl}_{2}(10 \mathrm{~mL} \times 3)$, and the combined organic layers were dried over $\mathrm{MgSO}_{4}$ and concentrated. The resulting dark-brown oil was purified by column chromatography (hexanes $/ \mathrm{Et}_{2} \mathrm{O}$ 8:1), which furnished the title compound $(820 \mathrm{mg}, 66 \%)$. This reaction was not optimized.

${ }^{1} \mathrm{H}$ NMR $\left(\mathrm{CDCl}_{3}, 400 \mathrm{MHz}\right)$ 8 7.91-7.89 (m, 2H), $7.77(\mathrm{~d}, J=12.2 \mathrm{~Hz}, 1 \mathrm{H}), 7.54-7.52(\mathrm{~m}$, $1 \mathrm{H}), 7.50-7.44(\mathrm{~m}, 2 \mathrm{H}), 6.35(\mathrm{~d}, J=12.2 \mathrm{~Hz}, 1 \mathrm{H}), 4.13-4.09(\mathrm{~m}, 2 \mathrm{H}), 1.15-1.11(\mathrm{~m}, 2 \mathrm{H}), 0.09$ (s, 9H);

${ }^{13} \mathrm{C}$ NMR $\left(\mathrm{CDCl}_{3}, 100 \mathrm{MHz}\right) \delta$ 218.2, 191.0, 164.4, 132.4, 128.6, 128.2, 102.3, 70.5, 18.1, -1.2 ;

IR (film) 3064, 2953, 2896, 1664, 1601, 1586, 1575, 1447, 1381, 1250, 1192, 1172, 858, 838 $\mathrm{cm}^{-1}$;

LCMS (ES+APCI) calcd for $\mathrm{C}_{14} \mathrm{H}_{20} \mathrm{O}_{2} \mathrm{SiNa}\left(\mathrm{M}+\mathrm{Na}^{+}\right)$271.1125, found 271.2.

((2S,3R,5S)-3-(2-(Trimethylsilyl)ethoxy)-tetrahydro-5-phenylfuran-2-yl)methanol. General Procedure A was followed, with (-)-bpy*, (E)-3-(2-(trimethylsilyl)ethoxy)-1phenylprop-2-en-1-one (248 mg, $1.0 \mathrm{mmol})$, and 2,6-diisopropylphenyldiazoacetate (271 $\mathrm{mg}, 1.1 \mathrm{mmol}$ ). ${ }^{1} \mathrm{H}$ NMR analysis of the unpurified dihydrofuran product revealed only one diastereomer.

The unpurified product was subjected to hydrogenation (1 atm) with $\mathrm{Pd}$ on activated carbon $(10 \% \mathrm{w} / \mathrm{w} ; 5 \mathrm{mg})$ and $\mathrm{NEt}_{3}(70 \mu \mathrm{L}, 0.5 \mathrm{mmol})$ in $\mathrm{MeOH}(3.0 \mathrm{~mL})$ for 1 $\mathrm{h}$. The reaction mixture was filtered through a plug of silica gel $\left(\mathrm{Et}_{2} \mathrm{O}\right.$ as the eluant) and

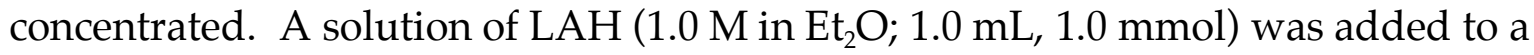
solution of the tetrahydrofuran in $\mathrm{Et}_{2} \mathrm{O}(4.0 \mathrm{~mL})$. The mixture was stirred for $5 \mathrm{~min}$, and then the reaction was quenched by adding water $(0.1 \mathrm{~mL})$ and stirring for $10 \mathrm{~min}$. The reaction mixture was dried over $\mathrm{MgSO}_{4}$, filtered, concentrated, and purified by column chromatography (hexanes $/ \mathrm{Et}_{2} \mathrm{O} 5: 1$ ), which furnished the title compound as a colorless oil: run 1, $216 \mathrm{mg}(73 \%)$; run $2,236 \mathrm{mg}(80 \%)$.

${ }^{1} \mathrm{H}$ NMR $\left(\mathrm{CDCl}_{3}, 400 \mathrm{MHz}\right)$ 8 7.37-7.28 (m, 5H), $5.12(\mathrm{dd}, J=10.5,5.4 \mathrm{~Hz}, 1 \mathrm{H}), 4.09$ $(\mathrm{dt}, J=4.8,3.4 \mathrm{~Hz}, 1 \mathrm{H}), 4.02(\mathrm{ddd}, J=6.3,2.8,1.4 \mathrm{~Hz}, 1 \mathrm{H}), 3.88(\mathrm{dd}, J=11.7,3.6 \mathrm{~Hz}, 1 \mathrm{H})$, $3.72(\mathrm{dd}, J=11.7,4.9 \mathrm{~Hz}, 1 \mathrm{H}), 3.60-3.53(\mathrm{~m}, 2 \mathrm{H}), 2.35(\mathrm{ddd}, J=13.3,5.4,1.5 \mathrm{~Hz}, 1 \mathrm{H}), 1.97$ (br s, $1 \mathrm{H}), 1.91(\mathrm{ddd}, J=13.3,10.5,6.4 \mathrm{~Hz}, 1 \mathrm{H}), 1.00-0.96(\mathrm{~m}, 2 \mathrm{H}), 0.05(\mathrm{~s}, 9 \mathrm{H})$;

${ }^{13} \mathrm{C} \mathrm{NMR}\left(\mathrm{CDCl}_{3}, 100 \mathrm{MHz}\right) \delta 141.6,128.9,128.2,126.4,85.6,81.1,80.8,67.0,64.2$, $41.7,18.8,-0.9$; 
IR (film) 3414, 2952, 2893, 1715, 1362, 1222, 1098, $1057 \mathrm{~cm}^{-1}$;

LCMS (ES+APCI) calcd for $\mathrm{C}_{16} \mathrm{H}_{26} \mathrm{O}_{3} \mathrm{SiNa}\left(\mathrm{M}+\mathrm{Na}^{+}\right)$317.1543, found 317.2.

(2S,3R,5S)-Tetrahydro-2-(hydroxymethyl)-5-phenylfuran-3-ol. To a solution of ((2S,3R,5S)-3-(2-(trimethylsilyl)ethoxy)-tetrahydro-5-phenylfuran-2-yl)methanol (50.0 $\mathrm{mg}, 0.17 \mathrm{mmol})$ in toluene $(2.5 \mathrm{~mL})$ was added $\mathrm{BF}_{3} \cdot \mathrm{OEt}_{2}(23.5 \mu \mathrm{L}, 0.19 \mathrm{mmol})$. The reaction mixture was stirred for $1 \mathrm{~h}$, and then the reaction was quenched by the addition of a saturated solution of $\mathrm{NaHCO}_{3}(2.0 \mathrm{~mL})$. The mixture was extracted with $\mathrm{Et}_{2} \mathrm{O}(5 \mathrm{~mL} \times 3)$, dried over $\mathrm{MgSO}_{4}$, and concentrated. Purification by column chromatography $\left(\mathrm{CH}_{2} \mathrm{Cl}_{2} / \mathrm{MeOH}\right.$ 50:1) furnished the title compound as a white solid: run 1, $30 \mathrm{mg}$ (87\%; 93\% ee); run 2, $28 \mathrm{mg}(85 \% ; 94 \%$ ee). The ee was determined on an AD-H column (hexanes/iso-propanol 95:5, flow $1.0 \mathrm{~mL} / \mathrm{min}$ ) with $\mathrm{t}_{\mathrm{r}}$ (major) $32.1 \mathrm{~min}$, $\mathrm{t}_{\mathrm{r}}$ (minor) $28.9 \mathrm{~min}$.

The spectral data matched the values reported previously. ${ }^{3}$

$[\alpha]^{20}{ }_{\mathrm{D}}=-44.9\left(\mathrm{c}=1.00, \mathrm{CHCl}_{3}\right)$;

${ }^{1} \mathrm{H}$ NMR $\left(\mathrm{CDCl}_{3}, 400 \mathrm{MHz}\right) \delta 7.36-7.28(\mathrm{~m}, 5 \mathrm{H}), 5.18(\mathrm{dd}, J=10.2,5.6 \mathrm{~Hz}, 1 \mathrm{H}), 4.41$ $(\mathrm{dt}, J=6.0,2.4 \mathrm{~Hz}, 1 \mathrm{H}), 4.03-3.99(\mathrm{~m}, 1 \mathrm{H}), 3.81(\mathrm{dd}, J=11.6,4.3 \mathrm{~Hz}, 1 \mathrm{H}), 3.73(\mathrm{dd}, J=$ 11.6, $5.0 \mathrm{~Hz}, 1 \mathrm{H}), 2.37$ (br s, 2H), 2.25 (ddd, $J=13.3,5.7,1.9 \mathrm{~Hz}, 1 \mathrm{H}), 2.25$ (ddd, $J=13.3$, $10.2,6.3 \mathrm{~Hz}, 1 \mathrm{H})$;

${ }^{13} \mathrm{C}$ NMR $\left(\mathrm{CDCl}_{3}, 100 \mathrm{MHz}\right) \delta$ 141.2, 128.7, 128.1, 126.3, 87.5, 80.4, 73.9, 63.6, 44.0;

IR (film) 3274, 2940, 2832, 1490, 1452, 1358, 1102, 1054, $1028 \mathrm{~cm}^{-1}$;

LCMS (ES+APCI) calcd for $\mathrm{C}_{11} \mathrm{H}_{14} \mathrm{O}_{3} \mathrm{Na}\left(\mathrm{M}+\mathrm{Na}^{+}\right)$217.0835, found 217.1. 


\section{Determination of Stereochemistry}

The stereochemistry of the dihydrofurans was assigned on the basis of the synthesis described in Section 5 (above) and by correlation with (2R,3R)-3-methyl-5phenylpentane-1,2-diol.

$$
[\alpha]_{\mathrm{D}}^{20}=17.3\left(\mathrm{c}=0.83, \mathrm{CHCl}_{3}\right) .{ }_{\substack{{ }^{2} \\ \text { r.t. }}}^{\frac{1.0 \% \text { CuOTf}}{\mathrm{CH}_{2} \mathrm{Cl}_{2}}}
$$




\section{References}

(1) (a) Nicewicz, D. A.; Johnson, J. S. J. Am. Chem. Soc. 2005, 127, 6170-6171. (b) Doyle, M. P.; Austin, R. E.; Bailey, A. S.; Dwyer, M. P.; Dyatkin, A. B.; Kalinin, A. V.;

Kwan, M. M. Y.; Liras, S.; Oalmann, C. J.; Pieters, R. J.; Protopopova, M. N.; Raab, C. E.; Roos, G. H. P.; Zhou, Q.-L.; Martin, S. F. J. Am. Chem. Soc. 1995, 117, 5763-5775.

(2) Rios, R.; Liang, J.; Lo, M. M.-C.; Fu, G. C. Chem. Commun. 2000, 377-378.

(3) (a) Calter, M. A.; Zhu, C. J. Org. Chem. 1999, 64, 1415-1419. (b) Sas, B.; Van Hemel, J.; Vandenkerckhove, J.; Peys, E.; Van Der Eycken, J.; Van Hoof, S. U.S. Pat. Appl. 2004-760647, November 11, 2004.

(4) Barrow, R. A.; Hemscheidt, T.; Liang, J.; Paik, S.; Moore, R. E.; Tius, M. A. J. Am. Chem. Soc. 1995, 117, 2479-2490. 